\title{
Design and Implementation of a GPS Signal Simulator and Storage-and-Forward Machine
}

\author{
Hong Lin ${ }^{\text {a }}$, Wenfei Gong ${ }^{\mathrm{b}}$ \\ School of Electronic and Information Engineering, Beijing Jiaotong University, Beijing 100044, \\ China; \\ a15120016@bjtu.edu.cn, bwfgong@bjtu.edu.cn
}

Keywords: Signal simulation, storage-and-forward, FPGA, USB3.0.

\begin{abstract}
Currently, the ubiquitous application of navigation receivers prompts the development of signal simulators which can be used to test the performances of such receivers in various conditions. But tradition simulators cannot really restore the satellites' circulating conditions, which is not persuasive enough. This article aims to present a new method of generating navigation signals based on actual navigation signals, which can reconstruct the satellite constellations. This article provides not only the principles of the simulator but also the details of the design and implementation of the software simulator and a storage-and-forward machine which can collect GPS signals and play them back.
\end{abstract}

\section{Introduction}

Nowadays more and more efforts are put into researching and producing GNSS-dependent products. The development and test of such products may be difficult because the orbit that the satellite circulates and the path that the signal transmits are very complicated, which may be influenced by time, weather, satellites' status and other factors. Only real satellites' signals being used in the test can be persuasive enough on occasion that high precise test is required.

Currently, there are two problems to be solved in the process of testing the kinematic performances of a navigation products:

The real GPS signal data achieved cannot be put into use directly, because parameters of these data are usually stationary. Besides, testing the products outdoors in the real scene is limited by the weather and environment;

Satellite constellation is difficult to simulate

This article aims at proposing a new method of signal simulating, which extracts the useful information from real GPS signal and combine it with software simulating to reconstruct a kinematic environment where the tested receiver moves.

\section{Design and Implementation of Software Simulator}

\subsection{The Theory of Signal Simulation}

The signal of GPS at L1 frequency point is as follows ${ }^{[1]}$ :

$$
\operatorname{Signal}(\mathrm{t})=\sum_{i}^{N} \sqrt{2 P_{i}} D_{i}(t) C A_{i}(\mathrm{t}) \cos \left(2 \pi f t+\varphi_{i}\right)
$$

Where $P_{i}$ is power of signal; $D_{i}(t)$ is navigation message that visible satellites broadcast; $C A_{i}(t)$ is coarse acquisition code of satellite; $\cos \left(2 \pi f t+\varphi_{i}\right)$ is carrier; $\mathrm{N}$ is the number of visible satellites, which ranges from 4 to 12 .

This model is too simplified but shows the basic steps of simulating a GPS signal:

- Amplitude calculation

- Navigation message generation

- $\mathrm{C} / \mathrm{A}$ code generation

- Carrier generation and phase calculation 
Amplitude is determined by the Carrier Noise Rate (CNR) that users put in. The relation between CNR and Signal Noise Rate (SNR) can be described by the following equation ${ }^{[2]}$ :

Where BW is signal's bandwidth.

$$
\mathrm{CNR}=\mathrm{SNR} \times \mathrm{BW}
$$

Then compute signal's amplitude by the following equations:

$$
\begin{aligned}
& P_{\text {noise }}=B W \times \rho_{\text {noise }} \\
& P_{j}=S N R \times P_{\text {noise }} \\
& A_{j}(t)=\sqrt{2 P_{j}}
\end{aligned}
$$

For the precise simulation, the impact of time and environment should be taken into consideration. Therefore, a more complicated but more accurate model is set up as follows ${ }^{[3]}$ :

$$
\begin{aligned}
\operatorname{Signal}(\mathrm{t})= & \sum_{+n(t)+S_{M P}^{j}(t)} A_{j}(t) C A_{j}\left(t-\tau_{\text {ion }}^{j}-\tau_{d}^{j}\right) \cos \left[\omega_{L 1}\left(t+\tau_{\text {ion }}^{j}-\tau_{d}^{j}\right)\right] \\
& +n\left(\tau^{j}\right)
\end{aligned}
$$

Where $\tau_{i \Omega m}^{j}$ is ionospheric delay; $\tau_{d}^{j}$ is the delay caused by all the factors except ionospheric effect; $\mathrm{n}(\mathrm{t})$ is noise; $S_{M P}^{j}(t)$ is effect of multipath;

$\tau_{d}^{j}$ can be expressed as:

$$
\tau_{d}^{j}=\frac{R_{f}}{\epsilon}+\tau_{S V}^{j}+\tau_{\text {trop }}^{j}+\tau_{e p h}^{j}
$$

Where $R_{j}$ is the pseudo-range between GPS satellite and receiver; $\mathrm{c}$ is the speed of light; $\tau_{S V}^{j}$ is satellite clock error; $\tau_{\text {trop }}^{j}$ is troposphere delay; $\tau_{e p h}^{j}$ is ephemeris error.

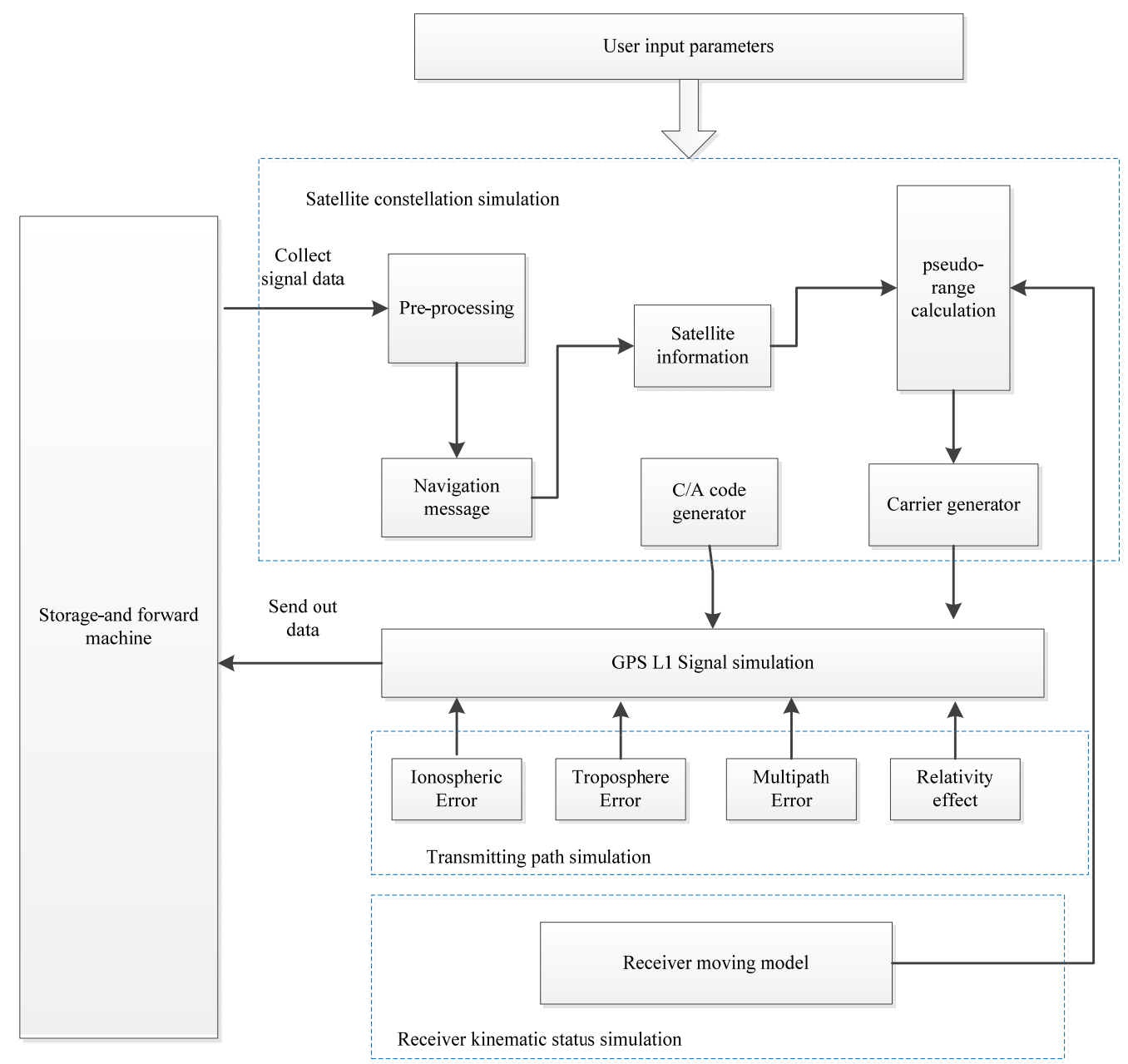

Figure 1. process of signal simulation

As figure 1 manifests, the simulator is comprised of two parts: the storage-and-forward machine which is based on FPGA and USB3.0, and the software simulator. 


\subsection{Design and Implementation}

The signal simulation process can be divided into three parts: satellite constellation simulation, transmitting path simulation and receiver kinematic status simulation.

The first part includes navigation message, C/A code and carrier simulation. Navigation message contains all the information of the satellite. C/A code is the ID of each satellite which provides the frequency shift because of its characteristics of autocorrelation. And the carrier carries the messages of immediate frequency, frequency shift and pseudo-range.

The C/A code and carrier is easy to generate while the navigation message is not because it contains lots of important information of satellite such as satellites' position, velocity and transmitting time. The traditional way to generate navigation data is to extract navigation information from the compiled ephemeris files and rewrite them as navigation message bits. The method adopted in this article is the opposite process, which is to remove the real GPS signal's C/A code and carrier and get navigation message bits and extract the satellite's information to reconstruct the ephemeris and calculate pseudo-range.

It is a new method of high efficiency. The process of obtaining navigation message is largely simplified because the step of acquisition and tracking can peel C/A code and carrier. After the processing of software receiver, navigation message can be saved as fixed form as Figure 2 shows:

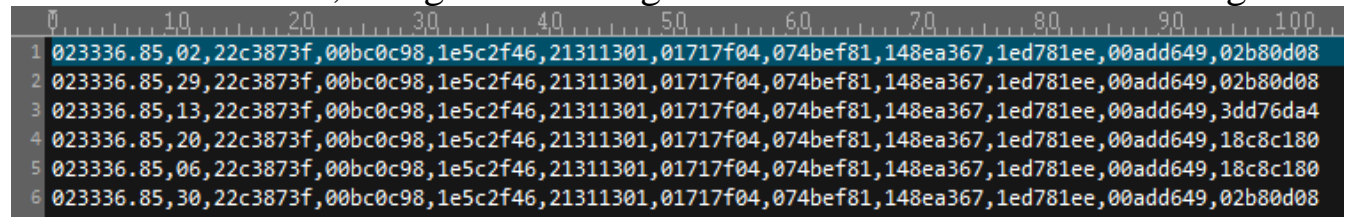

Figure 2. navigation message

In Figure 2, the first parameter is time of week, which is provided as a reference. The second parameter is the number of satellite which is followed by one of its sub-frame data. Each one frame of navigation message contains five sub-frames, and each sub-frame is made up with ten words, each of which has 24 data bits and 6 parity bits. The fourth and fifth sub-frames are paging. A suit of completed navigation message has 25 pages, which lasts for 12.5 minutes.

Traditional way of simulating constellation is to generate all the satellites information and estimate which satellite is visible according to its elevation. The advantage of this new method is that only the visible satellite is considered and there is no need to calculate the elevation and make a judgment. Besides, it is not necessary to analyze all the arguments.

Satellite's information should be extracted from the navigation message to calculate the satellite's position and velocity and other error parameters. The parameters included in the navigation message $^{[10]}$ is listed as table 1: 
Table 1. Ephemeris Parameters

\begin{tabular}{|c|c|c|c|}
\hline Parameter & Definition & $\begin{array}{c}\text { Bit } \\
\text { number(bit) }\end{array}$ & Unit \\
\hline$C_{r s}$ & Amplitude of sine correction to orbital radius & 16 & $\mathrm{~m}$ \\
\hline$\Delta n$ & Mean motion correction & 16 & $\pi / s$ \\
\hline$M_{0}$ & Mean anomaly(at Toe) & 32 & $\pi$ \\
\hline$C_{u c}$ & $\begin{array}{l}\text { Amplitude of cosine correction to argument of } \\
\text { latitude }\end{array}$ & 16 & rad \\
\hline$e$ & Eccentricity & 32 & none \\
\hline$C_{\text {us }}$ & $\begin{array}{l}\text { Amplitude of sine correction to argument of } \\
\text { latitude }\end{array}$ & 16 & $\mathrm{rad}$ \\
\hline$\sqrt{a}$ & Square root of semi-major axis & 32 & $m^{\frac{1}{2}}$ \\
\hline$t_{o e}$ & Reference time of ephemeris & 16 & $\mathrm{~s}$ \\
\hline$C_{i c}$ & $\begin{array}{l}\text { Amplitude of cosine correction to inclination } \\
\text { angle }\end{array}$ & 16 & rad \\
\hline$\Omega_{0}$ & $\begin{array}{l}\text { Longitude of the ascending node(at weekly } \\
\text { epoch) }\end{array}$ & 32 & rad \\
\hline$C_{i s}$ & $\begin{array}{c}\text { Amplitude of sine correction to inclination } \\
\text { angle }\end{array}$ & 16 & rad \\
\hline$i_{0}$ & Inclination angle(at Toe) & 32 & $\pi$ \\
\hline$C_{r c}$ & Amplitude of cosine correction to orbital radius & 16 & $\mathrm{~m}$ \\
\hline$\omega$ & Argument of perigee(at Toe) & 32 & $\pi$ \\
\hline$\dot{\mathrm{I}}$ & $\begin{array}{l}\text { Rage of change of longitude of the ascending } \\
\text { node }\end{array}$ & 24 & $\pi / s$ \\
\hline$\dot{\Omega}$ & Rate of change of inclination angle & 14 & $\pi / s$ \\
\hline
\end{tabular}

From the parameters above, the position of satellites can be calculated.

The position of satellites and receiver will be updated every 20 milliseconds, then re-compute the pseudo-range $\mathrm{R}(\mathrm{t})^{[11]}$ :

$$
\mathrm{R}(\mathrm{t})=\sqrt{\left(x^{t}-x\right)^{2}-\left(y^{t}-y\right)^{2}-\left(z^{t}-z\right)^{2}}+\delta t
$$

Thus, the pseudo-range carries not only the information of receiver's location but also its motion state.

\section{Design of the Storage-and-forward Machine}

This article also designs an immediate frequency signal storage-and-forward machine which has the functions of storing the collected signals and playing it back. 


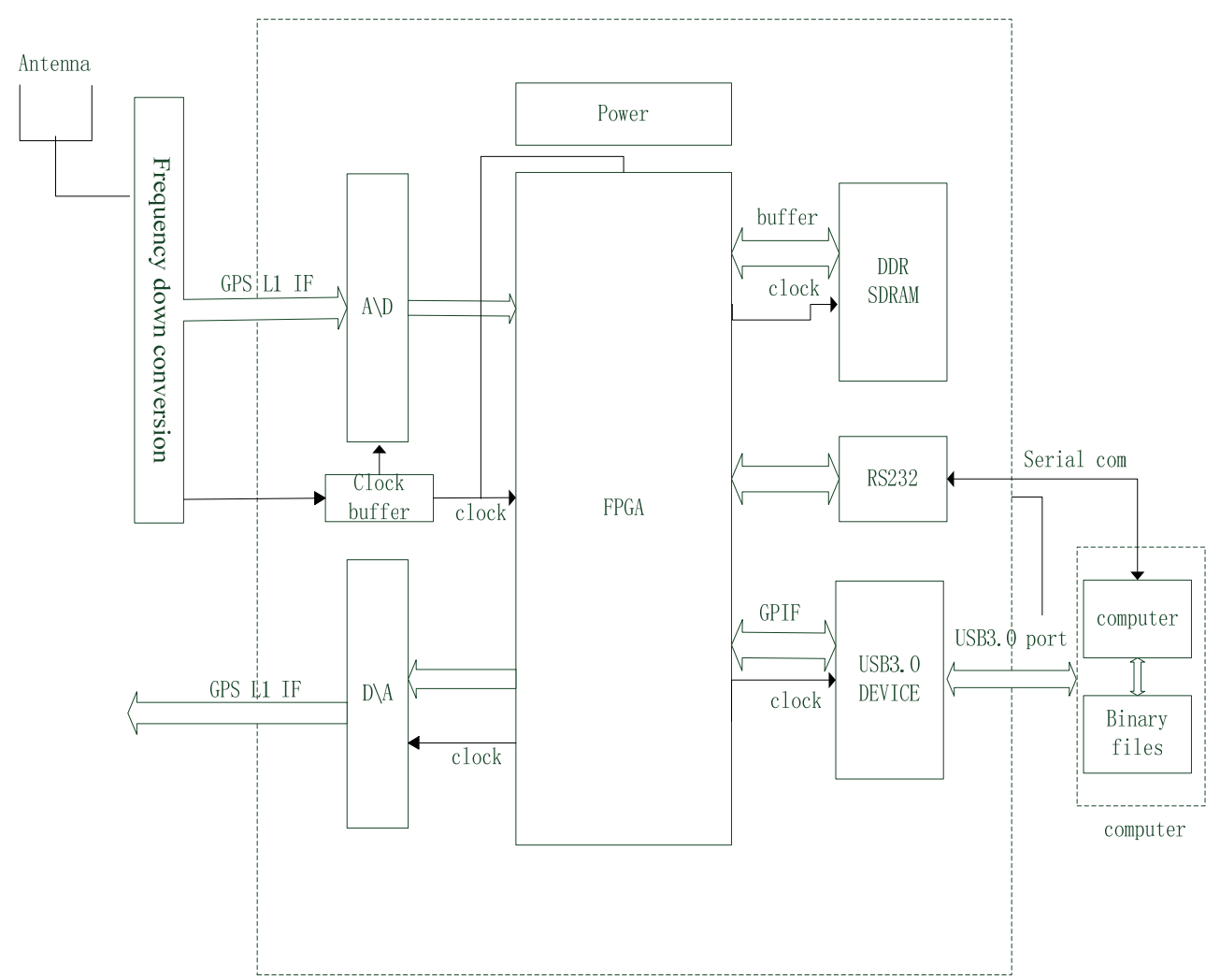

Figure 3. Design of storage-and-forward machine

As is shown in the figure 3, the storage-and-forward machine can collect real-time GPS signals, transmit the digital data that processed by ADC to computer by the aid of FPGA logic control and save the data as binary files. The data saved can put into various uses, such as satellite information analysis, ephemeris reconstruction and so on. The other way around is to read simulated data and send it to receivers to test the dynamic performances.

The storage-and-forward machine is modular design, which is composed of FPGA module, power module, clock buffer module, AD and DA module, DDR module and USB 3.0 module.

The upper computer sends the command of writing to USB device and the USB port is enabled, at the same time the FPGA come into the state of receiving GPS signal data. By means of FPGA logic control, ADC and external circuit are working. After the pre-processing, the GPS signals are converted to immediate frequency. A/D part transform the analog signals to digital data and output them to FPGA. Eventually, by the control of caching technique within FPGA, the data collected are send to USB 3.0 port and saved in the computer.

The preprocessing of converting RF to IF is necessary on ground that the circuit can handle with these signals that have lower frequency. However, considering the effects of Doppler frequency shift, the immediate frequency signal instead of baseband signal is the best choice.

In this part, much attention should be paid to the match of speed of FPGA transmitting data and computer saving data. Because the speed of transmitting data is higher than ordinary mechanical hard disk writing speed, only solid state drive can satisfy the need.

The core step is to send the simulated signals to receivers via this machine. The upper computer gives order to FPGA, and FPGA gets ready to read simulated data from USB 3.0 port. Then the DA is initialized and convert digital data to analog signals at constant speed by the technique of caching.

\section{Verification of Simulated Data}

To verify practical performance of this simulator, relative experiments are carried out.

First of all, the validity of satellite's signal acquired should be tested. Figure 4 shows the result of acquiring. 


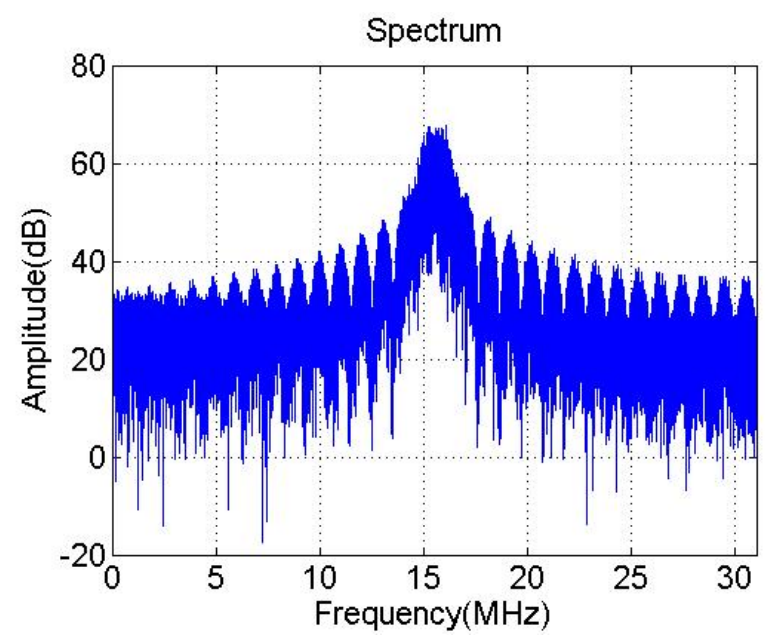

Figure 4. spectrum of aquired data

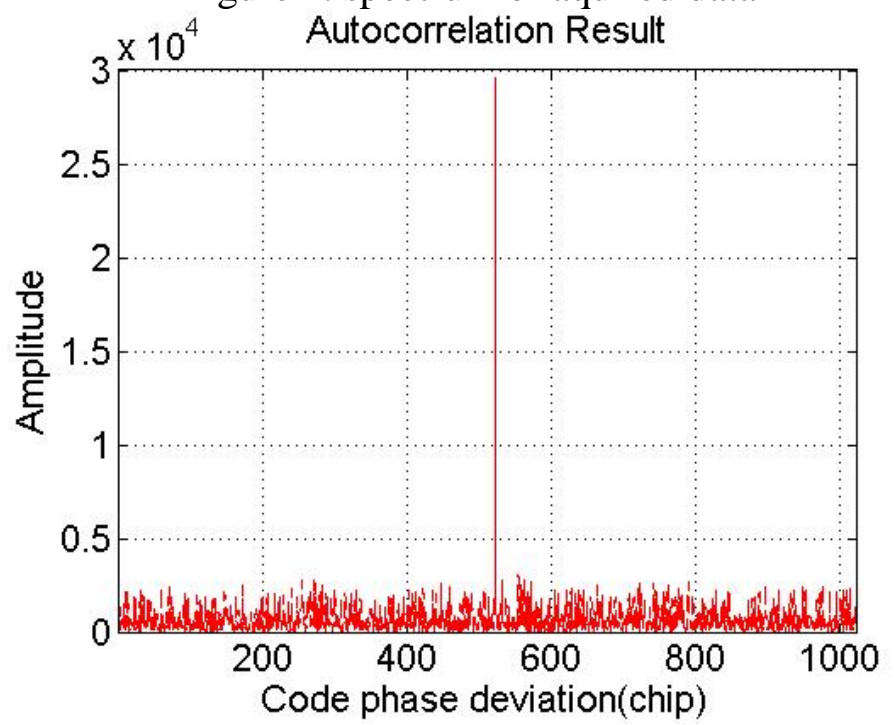

Figure 5. Autocorrelation result

Because of the characteristic of autocorrelation of C/A code, if the local C/A code and the received $\mathrm{C} / \mathrm{A}$ code has no phase deviation, there will be a pulse value after the processing of autocorrelation. The peak value in the figure 5 indicates that the data collected is GPS signal.

The core of the verification is to check out whether the simulated signals is valid or not. Figure 6 shows the result of the experiment that the receiver demodulates the simulated signals. 


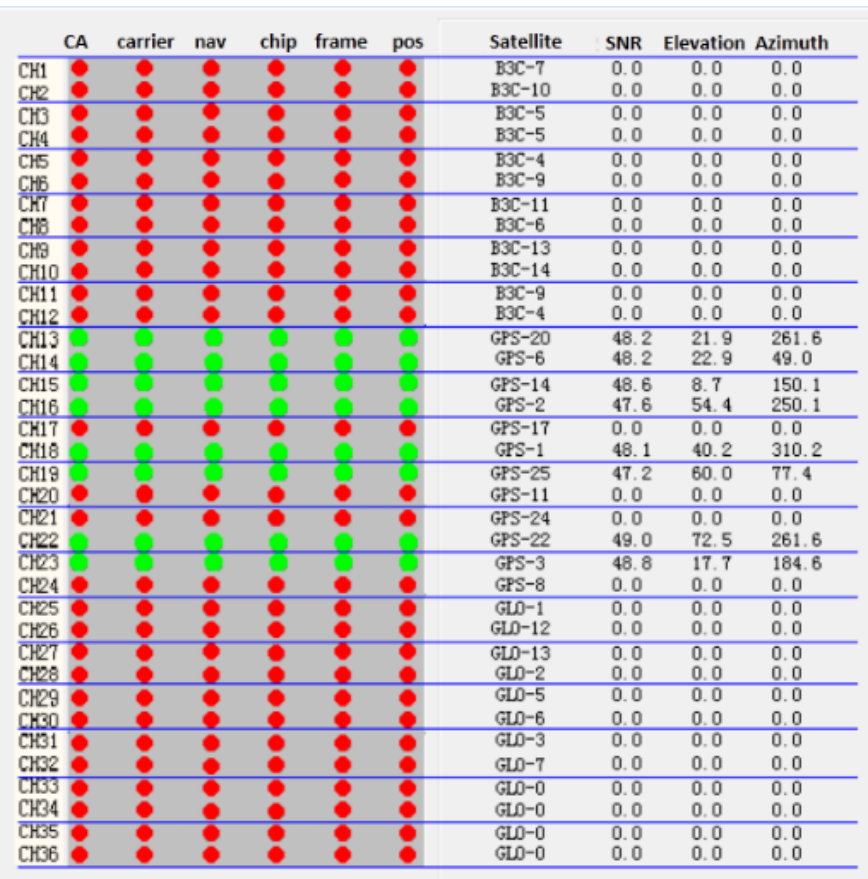

Figure 6. Result of positioning

In figure 6, the six lights present recognizing C/A code, carrier, navigation message, frequency shift, sub-frame head and positioning. The red light of certain whole line turning to green means that relative satellite can take part in positioning. Only when at least 4 satellites take part in positioning simultaneously can the receiver compute the location. Figure 6 indicates that there are 6 satellites take part in positioning, and relative SNR, elevation and azimuth angle are shown.

\section{Conclusion}

This article presents a new method of GPS signal simulation which combines the real GPS signals and receiver's dynamic model together. By the aid of storage-and-forward machine, real satellites' navigation messages can be recorded and modulated to generate direct spreading sequence. This article also provides the detailed process of implementation of the simulator which can increase testing efficiency to a great extent and reduce development cycle.

\section{References}

[1]. Elliot D Kaplan \& Christopher J Hegarty, “Understanding GPS Principles and applications”, Artech House,pp.142-150,2005

[2]. Xie Gang, “Principles of GPS and Receiver Design, Beijing”,pp.16-39,2009

[3]. Wang Yin. "Research of Algorithm of GPS Signal Simulation in Complicated Environment”. Nanjing University of Aeronautics and Astronautics, 2012.

[4]. Xu Jian, "Design of a Multi-bit ADC”,Shanghai Jiaotong University,2005

[5]. Jiang Wei,"GPS IF Signal Simulator Development and Research”, Harbin Institute of Technology,2009

[6]. Shang Guanwei, Liu Zhenguo, "GPS Intermediate Frequency Signal Simulation and Software Receiver Design”, Journal of the China Railway Society,pp.57-63, 2012

[7]. Zhang Wei,Zhang Ke,Xu Xizong, "Simulation Analysis of in GPS Signal C/A code Algorithm Design”, Communications Technology,pp.216-218, 2008

[8]. Li Chengjun, Lu Mingquan, Feng Zhenming, "Mathematical model and realization for GPS IF signal simulator”, Journal of Tsinghua University ( Sci \& Tech ),pp.1583-1585,2008

[9]. Li Qiushi, Yao Zheng, Lu Mingquan, "Design and Optimization of GPU-Based Real-time Software GNSS Signal Simulator”, Computer Simulation,pp.120-123,2013 
[10]. Bradford W.Parkinson, James J.Spilker.”Global Positioning System:Theory and Applications”,Washington D.C: American Institute of Aeronautics and Astronautics Inc.,1996

[11]. Yang Zhizhuan, Hu Xiulin,Zhang Yunyu,”Software Simulator of Digitized IF GPS Signals Based on MATLAB/simulink",GNSS World of China, pp.10-13,2006 